Cochrane Database of Systematic Reviews

\title{
Cognitive rehabilitation for attention deficits following stroke
} (Review)

Loetscher T, Potter KJ, Wong D, das Nair R

Loetscher T, Potter KJ, Wong D, das Nair R.

Cognitive rehabilitation for attention deficits following stroke.

Cochrane Database of Systematic Reviews 2019, Issue 11. Art. No.: CD002842.

DOI: 10.1002/14651858.CD002842.pub3.

www.cochranelibrary.com 
[Intervention Review]

\section{Cognitive rehabilitation for attention deficits following stroke}

Tobias Loetscher ${ }^{1}$, Kristy-Jane Potter ${ }^{2}$, Dana Wong ${ }^{3}$, Roshan das Nair ${ }^{2}$

1School of Psychology, University of South Australia, Adelaide, Australia. 2Division of Psychiatry \& Applied Psychology, University of Nottingham, Nottingham, UK. ${ }^{3}$ School of Psychology and Public Health, La Trobe University, Melbourne, Australia

Contact address: Tobias Loetscher, School of Psychology, University of South Australia, GPO Box 2471, Adelaide, SA, 5001, Australia. tobias.loetscher@unisa.edu.au, tobias.loetscher@alumni.ethz.ch.

Editorial group: Cochrane Stroke Group

Publication status and date: New search for studies and content updated (no change to conclusions), published in Issue 11, 2019.

Citation: Loetscher T, Potter KJ, Wong D, das Nair R. Cognitive rehabilitation for attention deficits following stroke. Cochrane Database of Systematic Reviews 2019, Issue 11. Art. No.: CD002842. DOI: 10.1002/14651858.CD002842.pub3.

Copyright @ 2019 The Cochrane Collaboration. Published by John Wiley \& Sons, Ltd.

\section{A B S T R A C T}

\section{Background}

Many survivors of stroke report attentional impairments, such as diminished concentration and distractibility. However, the effectiveness of cognitive rehabilitation for improving these impairments is uncertain. This is an update of the Cochrane Review first published in 2000 and previously updated in 2013.

\section{Objectives}

To determine whether people receiving cognitive rehabilitation for attention problems 1. show better outcomes in their attentional functions than those given no treatment or treatment as usual, and 2. have a better functional recovery, in terms of independence in activities of daily living, mood, and quality of life, than those given no treatment or treatment as usual.

\section{Search methods}

We searched the Cochrane Stroke Group Trials Register, CENTRAL, MEDLINE, Embase, CINAHL, PsycINFO, PsycBITE, REHABDATA and ongoing trials registers up to February 2019. We screened reference lists and tracked citations using Scopus.

\section{Selection criteria}

We included controlled clinical trials (CCTs) and randomised controlled trials (RCTs) of cognitive rehabilitation for impairments of attention for people with stroke. We did not consider listening to music, meditation, yoga, or mindfulness to be a form of cognitive rehabilitation. We only considered trials that selected people with demonstrable or self-reported attentional deficits. The primary outcomes were measures of global attentional functions, and secondary outcomes were measures of attentional domains (i.e. alertness, selective attention, sustained attention, divided attention), functional abilities, mood, and quality of life.

\section{Data collection and analysis}

Two review authors independently selected trials, extracted data, and assessed the risk of bias. We used the GRADE approach to assess the certainty of evidence for each outcome.

\section{Main results}

We included no new trials in this update. The results are unchanged from the previous review and are based on the data of six RCTs with 223 participants. All six RCTs compared cognitive rehabilitation with a usual care control.

Meta-analyses demonstrated no convincing effect of cognitive rehabilitation on subjective measures of attention either immediately after treatment (standardised mean difference (SMD) $0.53,95 \%$ confidence interval $(\mathrm{Cl})-0.03$ to $1.08 ; \mathrm{P}=0.06 ; 2$ studies, 53 participants; very low-quality evidence) or at follow-up (SMD $0.16,95 \% \mathrm{Cl}-0.23$ to $0.56 ; \mathrm{P}=0.41 ; 2$ studies, 99 participants; very low-quality evidence). 
People receiving cognitive rehabilitation (when compared with control) showed that measures of divided attention recorded immediately after treatment may improve (SMD 0.67,95\% Cl 0.35 to 0.98; $\mathrm{P}<0.0001 ; 4$ studies, 165 participants; low-quality evidence), but it is uncertain that these effects persisted (SMD $0.36,95 \% \mathrm{Cl}-0.04$ to $0.76 ; \mathrm{P}=0.08 ; 2$ studies, 99 participants; very low-quality evidence). There was no evidence for immediate or persistent effects of cognitive rehabilitation on alertness, selective attention, and sustained attention.

There was no convincing evidence for immediate or long-term effects of cognitive rehabilitation for attentional problems on functional abilities, mood, and quality of life after stroke.

\section{Authors' conclusions}

The effectiveness of cognitive rehabilitation for attention deficits following stroke remains unconfirmed. The results suggest there may be an immediate effect after treatment on attentional abilities, but future studies need to assess what helps this effect persist and generalise to attentional skills in daily life. Trials also need to have higher methodological quality and better reporting.

\section{PLAIN LANGUAGE SUMMARY}

\section{Cognitive rehabilitation for attention problems following stroke}

\section{Review question}

Our aim was to review the evidence about the effect of cognitive rehabilitation on attention, the ability to perform daily activities, mood, and quality of life in people who have attention problems following a stroke.

\section{Background}

Many people have problems with attention after stroke. They are unable to concentrate for prolonged periods of time and are distractible, being unable to focus on a specific task in the presence of competing information. 'Cognitive rehabilitation' involves providing therapeutic activities to reduce the severity of cognitive problems, like attention, following damage to the brain. The benefit of cognitive rehabilitation for attention problems following stroke is unclear.

\section{Study characteristics}

We identified six studies that compared cognitive rehabilitation with a control group who received their usual care (but not cognitive rehabilitation) for people with attention problems following stroke. We did not consider listening to music, meditation, yoga, or mindfulness to be a form of cognitive rehabilitation. The six studies involved 223 participants who demonstrated attentional problems or reported having such problems following stroke. The evidence is current to February 2019.

\section{Key results}

We found no evidence that cognitive rehabilitation improved general (global) measures of attention. The group that received cognitive rehabilitation performed better than the control group on tasks that required people to divide attention. However, this benefit was only seen immediately after the rehabilitation period with no suggestion that the benefits persist for longer. There was no evidence to suggest that cognitive rehabilitation was beneficial for other types of attention problems, or daily life activities, mood, or quality of life. More research is needed.

\section{Certainty of the evidence}

The very low to moderate methodological quality of the studies identified, and the lack of studies means that we cannot draw firm conclusions about the effect of cognitive rehabilitation for attention following stroke. 